\title{
Type D personality is a predictor of poor emotional quality of life in primary care heart failure patients independent of depressive symptoms and New York Heart Association functional class
}

\author{
Susanne S. Pedersen • Christoph Herrmann-Lingen • \\ Peter de Jonge $\cdot$ Martin Scherer
}

Received: June 3, 2009/Accepted: November 7, 2009/Published online: November 24, 2009

(C) The Author(s) 2009. This article is published with open access at Springerlink.com

\begin{abstract}
Quality of life is an important patient-centered outcome and predictor of mortality in heart failure, but little is known about the role of personality as a determinant of quality of life in this patient group. We examined the influence of Type D personality (i.e., increased negative emotions paired with emotional non-expression) on quality of life in primary care heart failure patients, using a prospective study design. Heart failure patients $(n=251)$ recruited from 44 primary care practices in Germany completed standardized questionnaires at baseline and
\end{abstract}

S. S. Pedersen $(\varangle) \cdot$ P. de Jonge

CoRPS, Center of Research on Psychology in Somatic diseases, Department of Medical Psychology, Room P506, Tilburg University, Warandelaan 2, PO Box 90153, 5000 LE Tilburg, The Netherlands

e-mail: s.s.pedersen@uvt.nl

URL: http://www.tilburguniversity.nl/corps/

C. Herrmann-Lingen

Department of Psychosomatic Medicine and Psychotherapy,

University of Göttingen, Göttingen, Germany

P. de Jonge

Department of Internal Medicine, University Medical Center

Groningen, University of Groningen, Groningen, The

Netherlands

P. de Jonge

Department of Psychiatry, University Medical Center Groningen, University of Groningen, Groningen, The Netherlands

\section{Scherer}

Department of General Practice and Family Medicine, University of Göttingen, Göttingen, Germany

M. Scherer

Institute of Social Medicine, University of Lübeck, Lübeck, Germany
9 months. The prevalence of Type D was 31.9\%. Type D patients experienced poorer emotional $(P<.001)$ and physical quality of life $(P=.01)$ at baseline and 9 months compared to non-Type $\mathrm{D}$ patients. There was no significant change in emotional $(P=.78)$ nor physical quality of life $(P=.74)$ over time; neither the interaction for time by Type $\mathrm{D}$ for emotional $(P=.31)$ nor physical quality of life $(P=.91)$ was significant, indicating that Type $\mathrm{D}$ exerted a stable effect on quality of life over time. Adjusting for demographics, New York Heart Association functional class, and depressive symptoms, Type D remained an independent determinant of emotional $(P=.03)$ but not physical quality of life $(P=.29)$. Primary care heart failure patients with a Type D personality experienced poorer emotional but not physical quality of life compared to nonType D patients. Patients with this personality profile should be identified in primary care to see if their treatment is optimal, as both Type D and poor quality of life have been associated with increased morbidity and mortality.

Keywords Depressive symptoms · Heart failure · Quality of life - Type D personality

\section{Introduction}

Heart failure is a debilitating clinical syndrome with a poor prognosis despite considerable improvements in treatment options over the last decade (Hunt 2005). Dyspnoea, oedema, fluid retention, pulmonary congestion and fatigue comprise common symptoms of heart failure, and although not necessarily present at the same time within an individual patient they all have a detrimental influence on functional status and quality of life (Hunt 2005; Juenger et al. 2002; Masoudi et al. 2004). 
Quality of life has gained increased recognition as an important patient-centered outcome (Krumholz et al. 2005) and has been incorporated as a performance measure for evaluating the quality of clinical care in the Improving Continuous Cardiac Care (IC3) registry (http:// www.improvingcardiaccare.org/Documents/IC3v1_Program Summary.pdf), a recent initiative by the American College of Cardiology Foundation in the United States. Some studies indicate that heart failure patients may prefer better quality of life over prolonged survival (Spertus 2008). Poor quality of life, as assessed by patient self-report, has also been shown to predict prognosis, including mortality and rehospitalization in heart failure patients (Mommersteeg et al. 2009). Hence, for the management of patients in clinical practice, knowledge of the patient's quality of life and in particular the clinical and psychological profile of those patients who experience poor quality of life is important for secondary prevention. Such information may help identify patients who may need more aggressive medical treatment or adjunctive psychological intervention (Spertus 2008).

Previously, mood states, such as anxiety and depression, have been identified as important determinants of quality of life both in heart failure patients and in patients with coronary artery disease (Pedersen et al. 2006; Rector et al. 1987; Schiffer et al. 2008a, b). However, little attention has been paid to the role of personality factors in heart failure, with only two studies focusing on the impact of the distressed (Type D) personality (i.e., increased tendency to experience increased negative emotions paired with emotional non-expression due to fear of rejection by others) on quality of life in heart failure (Schiffer et al. 2008a, b, 2005). These studies were conducted in outpatients with heart failure rather than primary care heart failure patients, with one of the studies using a cross-sectional study design (Schiffer et al. 2005). Both studies showed that Type D was associated with poorer quality of life and health status, adjusting for demographic and clinical characteristics, including left ventricular dysfunction and New York Heart Association (NYHA) functional class (Schiffer et al. 2008a, b, 2005). In heart failure, Type D has also been related to increased levels of pro-inflammatory cytokines (Denollet et al. 2009a, b), which are markers of poor prognosis in heart failure (Dunlay et al. 2008), and with late (>6 months) mortality (Schiffer et al. 2009). Given that heart failure patients seen by their primary care physician may have a milder disease spectrum, it is important to investigate whether findings from outpatients seen by their cardiologist or heart failure nurse generalize to this patient group. In the general cardiovascular literature, there is accumulating evidence that Type D is a risk marker for adverse health outcomes across different forms of cardiovascular disease (Pedersen and Denollet 2006). Type D personality has also been shown to predict adverse health outcomes in 482 older primary care patients above and beyond personality traits encompassed in the Five Factor Model of personality, indicating that Type D provides unique risk that is not captured by standard personality traits (Chapman et al. 2007).

In the current study, we examined (1) the influence of Type D personality on quality of life at baseline and 9-month follow-up in primary care heart failure patients, and (2) the extent to which the influence of personality on quality of life is confounded by NYHA class and depressive symptoms.

\section{Methods}

\section{Patients and design}

All 44 general practices within a radius of $30 \mathrm{~km}$ around Göttingen, Germany, were selected for participation in this study. In these practices, electronic patient data (date of birth, sex, diagnosis) were exported via a defined interface and transferred to a database. This database was screened for patients with the documented diagnosis of heart failure using a specific search strategy based on structured standard query language. Subsequently, all general practitioners received lists with patient identification codes and dates of birth for re-identification of the patients in the electronic practice information system. Based on a predefined algorithm, general practitioners were asked to exclude patients from the lists if their diagnosis of heart failure was not valid, if patients had a life-threatening illness, cancer, severe disability, were unable to communicate (e.g., due to dementia), insufficiently proficient in the German language to complete questionnaires, had moved to a region outside the study area, or were only seen by locums (i.e., a temporary replacement physician).

Eligible patients were asked to complete a set of standardized and validated questionnaires at baseline and at 9 months, of whom 363 patients agreed. Information on patient recruitment and baseline characteristics of the sample have been published previously (Scherer et al. 2006). Of the 363 patients, 310 patients could be interviewed at 9 months (Fig. 1). For the current study, analyses were based on 251 patients, for whom it was possible to calculate a scale score for the questionnaires under study. Factors contributing to differences between patients included in the analyses $(n=251)$ and excluded from analyses $(n=59)$ were age and heart failure severity according to NYHA classification. Patients excluded from analyses were more likely to be older (mean age 77.5) and to have higher NYHA functional class at baseline (NYHA II-IV $55.7 \%$ ). 


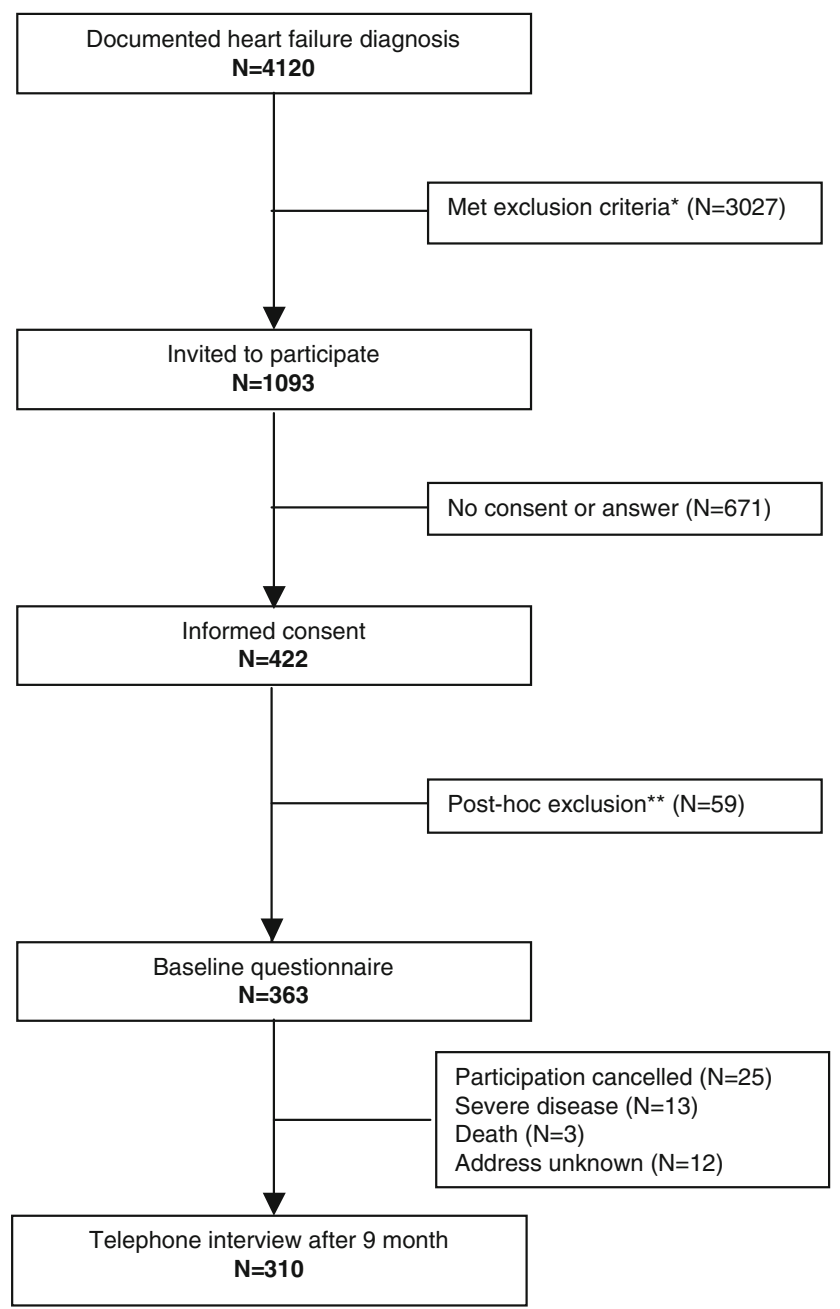

Fig. 1 Flow chart of study population. * No heart failure $(n=455)$; terminal illness $(n=24)$; death $(n=639)$; patients seen by locums $(n=469)$; other reasons $(n=1,440$ : export of electronic patient record incorrect (486), dementia (377), severe psychiatric disease (110) - the remaining patients (467) had either changed domicile or general practitioner or had an urgent need of care, inability to communicate or suffered from cancer). ** Consent to study participation was withdrawn (21), questionnaire not filled in (34), heart failure diagnosis not valid (2), communication problems (2)

The study protocol was approved by the ethics committee of the University of Göttingen, Germany. All patients provided written informed consent, and the study was carried out in accordance with the Helsinki Declaration.

\section{Materials}

\section{Demographic and clinical variables}

Demographic variables included gender, age, marital status, education, and employment status. Clinical variables comprised NYHA class, as a measure of the patient's functional status. Except demographic variables and the assessment of Type D personality and the depression subscale of the Hospital Anxiety and Depression Scale, all other assessments were performed at baseline as well as 9 months later.

\section{Quality of life}

Quality of life was assessed with the Minnesota Living with Heart Failure Questionnaire, which is a 21-item disease-specific measure (Rector 2005; Rector et al. 1987). Items are answered on a 6-point Likert scale from 0 (no) to 5 (very much). The total Minnesota Living with Heart Failure Questionnaire score ranges from 0 to 105, with a higher score indicating poorer quality of life. In addition to a total score, it is possible to derive an emotional (5 items; questions 17-21; score range 0-25) and physical dimension (8 items; questions $2-7,12-13$; score range $0-40$ ) of quality of life (de Rivas et al. 2008; Rector 2005; Schiffer et al. 2008a, b). A sample item of the emotional subscale is: "Making you feel you are a burden to your family or friends". A sample item of the physical subscale is: "Making your walking about or climbing stairs difficult". For the purpose of the current study we used the emotional and physical quality of life subscales rather than the total score, as they represent two different facets of quality of life, and it is possible that the predictors of poor emotional versus physical quality of life may be different. The Minnesota Living with Heart Failure Questionnaire is a psychometrically sound measure, with good internal consistency as measured by Cronbach's $\alpha=.91-.96$ for the total scale (Rector et al. 1987), and .94 and .88 for the physical and emotional dimension, respectively (Rector 2005). In the current study, Cronbach's $\alpha$ for the physical dimension of the Minnesota Living with Heart Failure Questionnaire was .91 and .82 for the emotional dimension.

\section{Type D personality}

The 14-item Type D Scale (DS14) was used to assess Type D personality (Denollet 2005). The Type D Scale measures two stable personality traits, that is negative affectivity (7 items; e.g. "I often feel unhappy" and "I am often irritated") and social inhibition (7 items; e.g. "I am a closed person" and "I find it hard to start a conversation") (Denollet 2005). All items are answered on a 5-point Likert scale from 0 (false) to 4 (true). Patients are categorized as Type D if they score $\geq 10$ on both subscales (Denollet 2005). Item-response theory has shown this cut-off to be the most optimal (Emons et al. 2007). The Type D Scale has good psychometric properties, with Cronbach's $\alpha=.88 / .86$ and 3-month test-retest reliability $r=.72 / .82$ for the negative affectivity and social inhibition subscales, respectively (Denollet 2005). The German version of the 
Type D Scale has been validated in a sample of 2,421 participants, including cardiac and psychosomatic patients and healthy factory workers, and found equivalent to the Flemish original version (Grande et al. 2004). In the current study, Crobach's $\alpha$ for negative affectivity was .82 and .83 for social inhibition. Type D is not confounded by disease severity, as measured by left ventricular ejection fraction (Martens et al. 2007) and NYHA class (Scherer et al. 2006), indicating that patients with this personality profile do not have more severe heart disease than nonType D patients. Type D personality also distinguishes itself from depression, in that it is a chronic rather than an episodic risk factor, and stipulates how patients deal with their negative emotions, due to the inclusion of the social inhibition component (Pedersen and Denollet 2006). The Type D scale has been listed among the recommended measures to use when screening for psychosocial risk factors in clinical cardiology practice in order to identify high-risk patients (Albus et al. 2004).

\section{Depressive symptoms}

The 7-item depression subscale of the Hospital Anxiety and Depression Scale was used to assess symptoms of depression (e.g. "I have lost interest in my appearance") (Zigmond and Snaith 1983). All items are answered on a 4-point Likert scale from 0 to 3, with the score range being 0-21. The Hospital Anxiety and Depression Scale is a valid and reliable instrument, as indicated in a recent review of 15 studies, with Cronbach's $\alpha$ ranging from .67 to .90 for the depression subscale (Bjelland et al. 2002). In the current study, a Cronbach's $\alpha$ of .84 was found. A cut-off $\geq 8$ is used to indicate probable clinical levels of depression, with sensitivity and specificity ranging between .70 and .90 for most reviewed studies (Bjelland et al. 2002). The Hospital Anxiety and Depression Scale has also been shown to predict mortality in patients referred for exercise testing (Herrmann et al. 2000) and in heart failure patients (Junger et al. 2005). Although the Hospital Anxiety and Depression Scale is devoid of somatic symptoms and hence would be expected to be unconfounded by symptoms of somatic disease, we have previously shown that Hospital Anxiety and Depression Scale scores increase with higher NYHA class in heart failure patients (Scherer et al. 2006).

\section{Statistical analyses}

All data were analyzed using SPSS 17.0 for Windows (SPSS Inc., Chicago, Illinois). Prior to statistical analyses, we dichotomized NYHA class into NYHA I versus II-IV, as only 26 patients were in class III and 4 patients in class IV. For comparisons of Type D with non-Type D patients on baseline characteristics, we used the Chi-square test (Fisher's exact text when appropriate) for nominal variables and Student's $t$-test for continuous variables. The Pearson correlation coefficient was used to determine the correlation between the Type D subcomponents negative affectivity and social inhibition and quality of life at both time points, whereas Spearman's rho was used to evaluate the correlation between Type D caseness and quality of life. These relationships between the independent variable (i.e., Type D) and the dependent variable (i.e., quality of life) were investigated prior to further analyses, in order to rule out that Type D would predict quality of life outcomes solely due to there being too large an overlap between the two constructs. Analysis of variance with repeated measures was used to examine changes in quality of life during the 9-month follow-up period and the influence of Type D personality on quality of life. Analysis of covariance was used to adjust the influence of Type D on quality of life for potential confounders; a priori based on the literature, we had decided to include gender, age, marital status, education, employment status, NYHA class, and depressive symptoms as covariates in this analysis. All tests were twotailed, and a $P$-value $<.05$ was used to indicate statistical significance.

\section{Results}

Baseline characteristics of the sample

The prevalence of Type D personality was $31.9 \%$. Baseline characteristics for the total sample and stratified by Type D personality are shown in Table 1 . Heart failure patients with a Type $\mathrm{D}$ personality disposition were slightly younger and experienced more depressive symptoms compared to non-Type D patients. No other statistically significant differences were found on baseline characteristics between the two groups.

Extent of independence between the constructs Type D personality and quality of life

In order to rule out that Type D would predict quality of life outcomes solely due to there being too large an overlap between the two constructs, we first investigated the relationship between Type D and quality of life using correlational analyses. Pearson's correlations showed that the Type D Scale negative affectivity component correlated significantly with emotional $(r=.53 ; P<.001)$ and physical quality of life $(r=.40 ; P<.001)$ at baseline and also with emotional $(r=.50 ; P<.001)$ and physical quality of life $(r=.38 ; P<.001)$ at 9 months. Similarly, 
Table 1 Baseline characteristics for the total sample and stratified by Type D personality

\begin{tabular}{|c|c|c|c|c|}
\hline & $\begin{array}{l}\text { Total } \\
(n=251)\end{array}$ & $\begin{array}{l}\text { Type D } \\
(n=80)\end{array}$ & $\begin{array}{l}\text { Non-Type D } \\
(n=171)\end{array}$ & $P$ \\
\hline Age, mean (SD) & $71.9(9.4)$ & $70.0(9.8)$ & $72.8(9.0)$ & .03 \\
\hline Male gender & $47.4 \%$ & $48.8 \%$ & $46.8 \%$ & .88 \\
\hline Living with a partner & $69.6 \%$ & $65.0 \%$ & $71.8 \%$ & .35 \\
\hline Low education ${ }^{\mathrm{a}}$ & $73.6 \%$ & $72.5 \%$ & $74.1 \%$ & .91 \\
\hline Employed & $10.0 \%$ & $11.8 \%$ & $9.6 \%$ & .77 \\
\hline NYHA class II-IV & $42.6 \%$ & $41.3 \%$ & $43.3 \%$ & .87 \\
\hline Depressive symptoms & $21.5 \%$ & $43.8 \%$ & $11.1 \%$ & $<.001$ \\
\hline
\end{tabular}

a $\leq 9$ years

Bold values are statistically significant at the designed cut-off of $P<.05$

the Type D Scale social inhibition component correlated significantly with emotional $(r=.27 ; P<.001)$ and physical quality of life $(r=.18 ; P=.004)$ at baseline and also with emotional $(r=.27 ; P<.001)$ and physical quality of life $(r=.23 ; P<.001)$ at 9 months. Given that a high score on the Minnesota Living with Heart Failure Questionnaire reflects a poorer quality of life, higher scores on the Type D Scale negative affectivity and social inhibition components were associated with poorer emotional and physical quality of life. Nevertheless, the shared variance between the subcomponents of the Type D Scale and the quality of life scores ranged from only 3 to $28 \%$, with none of the correlations exceeding the cut-off $>.85$ (Munro 2001). This indicates that there is not a problem with collinearity nor that there is a question of the argument being circular if Type D is related to quality of life in further analyses.

These results were confirmed when relating Type D caseness to quality of life scores, with the shared variance between Type D personality and quality of life being lower than when using continuous scores for the Type D subcomponents, although Type D was still significantly correlated with baseline emotional $(\rho=.28 ; P<.001)$ and physical quality of life $(\rho=.17 ; P=.009)$ and with 9-month emotional $(\rho=.22 ; P<.001)$ and physical quality of life $(\rho=.15 ; P<.02)$.

The influence of Type D personality on quality of life at baseline and 9 months (unadjusted)

Analysis of variance with repeated measures showed that Type D patients experienced poorer emotional quality of life both at baseline and 9 months compared to non-Type D patients $(F(1,249)=24.784 ; P<.001 ;$ partial eta-squared $=.091)$ (Table 2$)$. There was no significant change in emotional quality of life between baseline and 9 months $(F(1,249)=.076 ; P=.78$; partial etasquared $=.000$ ), nor was there a differential change in
Table 2 Quality of life at baseline and 9 months, stratified by Type D personality, presented as means (SDs)

\begin{tabular}{lll}
\hline & $\begin{array}{l}\text { Type D } \\
(n=80)\end{array}$ & $\begin{array}{l}\text { Non-Type D } \\
(n=171)\end{array}$ \\
\hline $\begin{array}{l}\text { Emotional quality of life } \\
\text { Baseline }\end{array}$ & $5.96(5.92)$ & $2.89(3.75)$ \\
9 months & $5.78(5.72)$ & $3.20(3.92)$ \\
Physical quality of life & & $12.99(10.27)$ \\
Baseline & $16.33(10.08)$ & $13.22(10.48)$ \\
9 months & $16.44(10.51)$ &
\end{tabular}

Analysis of variance with repeated measures; a high score indicates poor quality of life

emotional quality of life over time between Type $\mathrm{D}$ and nonType D patients, as indicated by the non-significant interaction effect for time by Type $\mathrm{D}(F(1,249)=1.043$; $P=.31 ;$ partial eta-squared $=.004)$. Hence, Type $\mathrm{D}$ exerted a stable effect on quality of life over time.

Similar results were found for the physical dimension of quality of life, with Type D patients experiencing poorer physical quality of life at both time points compared to nonType D patients $(F(1,249)=6.243 ; P=.01$; partial etasquared $=.024)($ Table 2$)$. There was also no change in physical quality of life over time $(F(1,249)=.115$; $P=.74$; partial eta-squared $=.000)$, nor was the interaction effect for time by Type D significant $(F(1,249)=.014$; $P=.91 ;$ partial eta-squared $=.000)$, indicating that Type $\mathrm{D}$ also exerted a stable effect on physical quality of life over time.

The influence of Type D personality on quality of life at baseline and 9 months (adjusted)

Type D was still a significant independent determinant of poor emotional quality of life $(F(1,227)=4.808 ; P=.03$; partial eta-squared $=.021$ ), adjusting for gender, age, marital status, education, employment status, NYHA class, 
and depressive symptoms, using analysis of covariance with repeated measures. Younger age $(F(1,227)=4.947$; $P=.03$; partial eta-squared $=.021)$, NYHA class II-IV $(F(1,227)=27.550 ; P<.001 ;$ partial eta-squared $=.108)$, and depressive symptoms $(F(1,227)=56.181 ; P<.001$; partial eta-squared $=.198$ ) were also significant determinants of poor emotional quality of life. In adjusted analysis, the within-subjects effect for time was still not significant $(F(1,227)=.810 ; P=.37 ;$ partial eta-squared $=.004)$, indicating that there was no change in emotional quality of life over time. None of the other interaction effects for time by covariates were significant ( $P$ 's $>.05$ ), except for NYHA class by time, with a plot of the estimated marginal means indicating that patients in NYHA class II-IV experienced a deterioration in emotional quality of life over time.

In adjusted analysis, Type D was no longer an independent determinant of poor physical quality of life $(F(1,227)=1.115 ; P=.29 ;$ partial eta-squared $=.005)$. The only covariates significantly associated with poor physical quality of life were female gender $(F(1,227)=$ $6.560 ; P=.01$; partial eta-squared $=.028)$, NYHA class IIIV $(F(1,227)=149.759 ; P<.001 ;$ partial eta-squared $=$ $.397)$, and depressive symptoms $(F(1,227)=25.085$; $P<.001$; partial eta-squared $=.100)$. In adjusted analysis, there was also no change in physical quality of life over time $(F(1,227)=.005 ; P=.94 ;$ partial eta-squared $=.000)$, nor were any of the within-subjects effects for time by covariates significant $\left(P^{\prime} \mathrm{s}>.05\right)$.

\section{Discussion}

In the current prospective study, primary care heart failure patients with a Type $\mathrm{D}$ personality experienced poorer emotional quality of life compared to non-Type D patients. The influence of personality on emotional quality of life in adjusted analysis could not be explained by demographic characteristics, NYHA class, and depressive symptoms. Although Type D personality was also associated with worse physical quality of life in unadjusted analysis, this relationship was no longer significant when controlling for demographic and clinical characteristics and depressive symptoms. NYHA class and depressive symptoms were also important, independent determinants of both poor emotional and physical quality of life.

Previous studies have shown that mood states, such as anxiety and depression, are important determinants of poor quality of life in heart failure patients, but these studies were either cross-sectional (Brenes 2007; Muller-Tasch et al. 2007) or conducted in outpatients with heart failure (Evangelista et al. 2008; Heo et al. 2008). The current study extends those findings by showing that personality factors add to the level of prediction of poor quality of life above and beyond depressive symptoms. This finding is consistent with a recent Dutch study of heart failure outpatients, showing that Type D patients report poorer emotional but not physical quality of life, as assessed by means of both disease-specific and generic measures (Schiffer et al. 2008a, b).

In the current study, we also found that higher NYHA class was associated with poorer quality of life. In previous studies, a worsening of functional status in heart failure outpatients predicted a decline in quality of life (Masoudi et al. 2004), and in a cross-sectional study of primary care heart failure NYHA functional class was associated with poor quality of life on several dimensions of the ShortForm Health Survey (SF-36) (Khunti et al. 2007). NYHA class III or IV has also been found to predict impairment in quality of life after open heart surgery (Pelle et al. 2009) and poor prognosis in heart failure (Andersson et al. 2008; Muntwyler et al. 2002). Despite the predictive value of NYHA class and its wide usage as a measure of functional status in heart failure patients, its objectivity and reproducibility are the subject of some debate (Raphael et al. 2007). There may also be some confounding between NYHA class and specific psychological measures, as we previously showed that Hospital Anxiety and Depression Scale scores, but not Type D personality, increased with higher NYHA class (Scherer et al. 2006).

The results of the current study have some implications for research and clinical practice. First, our results emphasize the importance of looking at the dimensions of quality of life (i.e., emotional versus physical) separately, as we only found a relationship between Type D personality and emotional quality of life, but not physical quality of life. This indicates that using a component or total score of quality of life may mask differences between subgroups on different aspects of quality of life. Second, previous studies in outpatients with heart failure and coronary artery disease have shown that Type D personality is associated with adverse health outcomes, including mortality, morbidity, and poor quality of life, independent of disease severity (Schiffer et al. 2008a, b, 2005; Pedersen and Denollet 2006; Denollet and Pedersen 2008). This was also confirmed in the current study, with patients with a Type D personality experiencing poorer emotional quality of life. To date, no randomized controlled trial targeting Type D personality has been conducted, but preliminary evidence suggests that these patients derive benefits from cardiac rehabilitation in terms of improved quality of life (Karlsson et al. 2007; Pelle et al. 2008). Hence, identification of this high-risk subgroup of patients seems worthwhile from a secondary prevention perspective, with respect to examining whether their heart failure is optimally managed or whether other treatment initiatives should be implemented. By analogy, we still do not know how to treat depression in 
cardiac patients, at least to the extent that such treatment leads to a concomitant improvement in survival (Berkman et al. 2003). However, this does not mean that we should not screen cardiac patients for depression nor that depression should be left untreated, as this would be unethical due to the known influence of depression on compliance, lifestyle modifications, participation in cardiac rehabilitation, mortality, and morbidity (Whooley 2009). Third, there are several advantages of using an instrument, such as the Type D scale, to identify patients with a general vulnerability to experience adverse health outcomes, over measures of anxiety, depression, and quality of life. These include the confounding of the latter measures with indices of somatic disease, such as NYHA class and left ventricular dysfunction. In a previous study, using the same patient sample as in the current study, we showed that a total score on the Hospital Anxiety and Depression Scale was confounded by NYHA class, whereas this was not the case for the Type D scale (Scherer et al. 2006). Similar confounding of the Beck Depression inventory, but not the Type D Scale, with indices of somatic disease has been found in patients with coronary artery disease (Martens et al. 2007). Moreover, evidence from patients with coronary artery disease indicates that not all distressed patients can be captured by standard measures of depression, and that despite some overlap Type D provides unique information above and beyond measures of depression. Of 1,205 myocardial infarction patients, $206(17 \%)$ patients had a clinical diagnosis of depression, $224(19 \%)$ patients a Type D personality, whereas only 90 (7\%) had both forms of distress (Denollet et al. 2009a, b). This may also be the case for quality of life, when used as a screening instrument rather than an outcome measure. Others have also indicated that measures that are sensitive as outcome measures may not necessarily be optimal screening instruments (Hevey et al. 2004). Furthermore, a recent study of patients with ischemic heart disease or heart failure indicated that despite some overlap between the Type D Scale and measures frequently used in cardiovascular research, including the Hospital Anxiety and Depression Scale, the Beck Depression Inventory, and the State-Trait Anxiety Inventory, the Type D Scale was the only examined measure that remained intact in its originally proposed form using factor analysis on item level and second-order level (Pelle et al. 2009).

The results of the current study have some limitations. First, given that the study was designed to examine predictors of patient-centered outcomes available in primary care practices, we had little information on traditional risk factors, such as co morbidity, hypercholesterolemia, left ventricular dysfunction, etc. Although we cannot rule out that left ventricular dysfunction may have confounded the results, evidence for an impact of left ventricular dysfunction on patient-centered outcomes, such as quality of life, anxiety and depressive symptoms, is mixed, with some (Bjelland et al. 2002; de Rivas et al. 2008) but not all studies (Lewis et al. 2007; Schiffer et al. 2008a, b, 2005) supporting such a relationship. Second, depressive symptoms were assessed by means of self-report rather than a clinical diagnostic interview. However, the Hospital Anxiety and Depression Scale has good sensitivity and specificity compared to the gold standard of a clinical diagnostic interview (Bjelland et al. 2002), and has also been shown to predict mortality in several cardiac patient groups (Herrmann et al. 2000; Junger et al. 2005). Third, patients excluded from analyses were more likely to be older and have a higher NYHA class compared to patients included in analyses, indicating that included patients had a less severe spectrum of disease, which might have led to a bias. Fourth, the results may primarily be generalizable to heart failure patients in NYHA class I and II, given that patients in NYHA class III and IV were under sampled. This necessitated the somewhat arbitrary cut-off of NYHA class I versus NYHA class II-IV for the statistical analyses. Fifth, several measures are available with which to assess quality of life of both a generic and disease-specific nature and a narrow or broad focus in terms of the number of domains of quality of life assessed. Although we chose to use the Minnesota Living with Heart Failure Questionnaire, which is a well accepted and frequently used disease-specific measure in heart failure, it only represents a limited number of quality of life domains. Sixth, it could be argued that the potential overlap between psychological factors and between psychological factors and quality of life may threaten the value of the results of the current study, as Type D might solely predict quality of life outcomes due to there being too large an overlap between the constructs. However, the shared variance between the subcomponents of the Type D Scale and the quality of life scores ranged from only 3 to $28 \%$, with none of the correlations exceeding the cut-off $>.85$, indicating that there was not a problem with collinearity (Munro 2001). Similarly, a recent study of patients with ischemic heart disease or heart failure examining the overlap between measures frequently used in cardiovascular research, including the Type D Scale and the Hospital Anxiety and Depression Scale, showed that these measures are distinct despite some overlap (Pelle et al. 2009). In the latter study, the Type D Scale was the only of the examined measures for which the original proposed structure remained intact using factor analysis on item level and second-order level.

Despite these limitations this study also has several strengths, including its prospective study design with the assessment of quality of life at two time points, the use of a disease-specific measure of quality of life, and the inclusion of personality factors, which is a novel approach 
in heart failure research. In addition, the study was conducted in a relatively large group of patients selected from the whole patient population of various practices by means of a pre-defined algorithm, and is therefore largely representative of the elderly heart failure patient seen in general practice. The relatively large number of exclusions can be traced back to this algorithm, making it unlikely that our sample was subject to an unintentional selection bias.

Further research is warranted to examine the added value of the Type D personality construct in primary care heart failure patients, particularly with respect to whether Type D affects mortality and rehospitalization in this patient group. Such research should aim to include more detailed information on the clinical characteristics of the patients than was available in the current study, in order to draw more firm conclusions. It would also be worthwhile to include other personality traits, such as comprised within the Five Factor Model of personality, to establish that Type D has added value above and beyond these traits as a predictor of health outcomes, as shown in older primary care patients (Chapman et al. 2007). Finally, further research on Type D should investigate whether the construct can best be viewed as a taxonomy, or whether patients in the grey zone around the cut-offs for determining Type D personality are also at risk for adverse health outcomes.

In conclusion, primary care heart failure patients with a Type D personality experienced poorer emotional but not physical quality of life compared to non-Type D patients. The influence of Type D personality on emotional quality of life could not be attributed to demographic characteristics, NYHA class, nor depressive symptoms. Patients with this personality profile should be identified in primary care, as both Type $\mathrm{D}$ and poor quality of life have been associated with mortality in this patient group. This could be done with the brief, validated Type D Scale (DS14), which comprises little burden to patients and to clinical practice. An international committee providing recommendations for the assessment of psychosocial risk factor in clinical cardiology practice has also recommended the use of the Type D Scale as one of the measures to identify highrisk patients (Albus et al. 2004).

Acknowledgments The study was supported by the German Ministry of Education and Research (BMBF), Grant Nr. 01 GK 0201.

Open Access This article is distributed under the terms of the Creative Commons Attribution Noncommercial License which permits any noncommercial use, distribution, and reproduction in any medium, provided the original author(s) and source are credited.

\section{References}

Albus, C., Jordan, J., \& Herrmann-Lingen, C. (2004). Screening for psychosocial risk factors in patients with coronary heart diseaserecommendations for clinical practice. European Journal of Cardiovascular Prevention and Rehabilitation, 11, 75-79.

Andersson, S. E., Edvinsson, M. L., Bjork, J., \& Edvinsson, L. (2008). High NT-proBNP is a strong predictor of outcome in elderly heart failure patients. American Journal of Geriatric Cardiology, $17,13-20$.

Berkman, L. F., Blumenthal, J., Burg, M., Carney, R. M., Catellier, D., Cowan, M. J., et al. (2003). Effects of treating depression and low perceived social support on clinical events after myocardial infarction: The Enhancing Recovery in Coronary Heart Disease Patients (ENRICHD) randomized trial. Journal of the American Medical Association, 289, 3106-3116.

Bjelland, I., Dahl, A. A., Haug, T. T., \& Neckelmann, D. (2002). The validity of the hospital anxiety and depression scale. An updated literature review. Journal of Psychosomatic Research, 52, 69-77.

Brenes, G. A. (2007). Anxiety, depression, and quality of life in primary care patients. Primary Care Companion to the Journal of Clinical Psychiatry, 9, 437-443.

Chapman, B. P., Duberstein, P. R., \& Lyness, J. M. (2007). The distressed personality type: Replicability and general health associations. European Journal of Personality, 21, 911-929.

de Rivas, B., Permanyer-Miralda, G., Brotons, C., Aznar, J., \& Sobreviela, E. (2008). Health-related quality of life in unselected outpatients with heart failure across Spain in two different health care levels. Magnitude and determinants of impairment: The INCA study. Quality of Life Research, 17, 1229-1238.

Denollet, J. (2005). DS14: Standard assessment of negative affectivity, social inhibition, and Type D personality. Psychosomatic Medicine, 67, 89-97.

Denollet, J., de Jonge, P., Kuyper, A., Schene, A. H., van Melle, J. P., Ormel, J., et al. (2009a). Depression and Type D personality represent different forms of distress in the Myocardial INfarction and Depression-Intervention Trial (MIND-IT). Psychological Medicine, 39, 749-756.

Denollet, J., \& Pedersen, S. S. (2008). Prognostic value of Type D personality compared with depressive symptoms. Archives of Internal Medicine, 168, 431-432.

Denollet, J., Schiffer, A. A., Kwaijtaal, M., Hooijkaas, H., Hendriks, E. H., Widdershoven, J. W., et al. (2009b). Usefulness of Type D personality and kidney dysfunction as predictors of interpatient variability in inflammatory activation in chronic heart failure. American Journal of Cardiology, 103, 399-404.

Dunlay, S. M., Weston, S. A., Redfield, M. M., Killian, J. M., \& Roger, V. L. (2008). Tumor necrosis factor-alpha and mortality in heart failure: A community study. Circulation, 118, 625-631.

Emons, W. H., Meijer, R. R., \& Denollet, J. (2007). Negative affectivity and social inhibition in cardiovascular disease: Evaluating Type D personality and its assessment using item response theory. Journal of Psychosomatic Research, 63, 27-39.

Evangelista, L. S., Moser, D. K., Westlake, C., Pike, N., TerGalstanyan, A., \& Dracup, K. (2008). Correlates of fatigue in patients with heart failure. Progress in Cardiovascular Nursing, $23,12-17$.

Grande, G., Jordan, J., Kümmel, M., Struwe, C., Schubmann, R., Schulze, F., et al. (2004). Evaluation of the German Type D Scale (DS14) and prevalence of the Type D personality pattern in cardiological and psychosomatic patients and healthy subjects. Psychotherapie, Psychosomatik en Medizinische Psychologie, 54, 413-422. 
Heo, S., Doering, L. V., Widener, J., \& Moser, D. K. (2008). Predictors and effect of physical symptom status on healthrelated quality of life in patients with heart failure. American Journal of Critical Care, 17, 124-132.

Herrmann, C., Brand-Driehorst, S., Buss, U., \& Ruger, U. (2000). Effects of anxiety and depression on 5-year mortality in 5, 057 patients referred for exercise testing. Journal of Psychosomatic Research, 48, 455-462.

Hevey, D., McGee, H. M., \& Horgan, J. (2004). Responsiveness of health-related quality of life outcome measures in cardiac rehabilitation: Comparison of cardiac rehabilitation outcome measures. Journal of Consulting and Clinical Psychology, 72, $1175-1180$.

Hunt, S. A. (2005). ACC/AHA 2005 guideline update for the diagnosis and management of chronic heart failure in the adult: A report of the American College of Cardiology/American Heart Association Task Force on Practice Guidelines (Writing Committee to Update the 2001 Guidelines for the Evaluation and Management of Heart Failure). Journal of the American College of Cardiology, 46, e1-e82.

Juenger, J., Schellberg, D., Kraemer, S., Haunstetter, A., Zugck, C., Herzog, W., et al. (2002). Health related quality of life in patients with congestive heart failure: Comparison with other chronic diseases and relation to functional variables. Heart, 87, 235-241.

Junger, J., Schellberg, D., Muller-Tasch, T., Raupp, G., Zugck, C., Haunstetter, A., et al. (2005). Depression increasingly predicts mortality in the course of congestive heart failure. European Journal of Heart Failure, 7, 261-267.

Karlsson, M. R., Edström-Plüss, C., Held, C., Henriksson, P., Billing, E., \& Wallén, N. H. (2007). Effects of expanded cardiac rehabilitation on psychosocial status in coronary artery disease with focus on type D characteristics. Journal of Behavioral Medicine, 30, 253-261.

Khunti, K., Stone, M., Paul, S., Baines, J., Gisborne, L., Farooqi, A., et al. (2007). Disease management programme for secondary prevention of coronary heart disease and heart failure in primary care: A cluster randomised controlled trial. Heart, 93, 13981405 .

Krumholz, H. M., Peterson, E. D., Ayanian, J. Z., Chin, M. H., DeBusk, R. F., Goldman, L., et al. (2005). Report of the National Heart, Lung, and Blood Institute working group on outcomes research in cardiovascular disease. Circulation, 111, 3158-3166.

Lewis, E. F., Lamas, G. A., O'Meara, E., Granger, C. B., Dunlap, M. E., McKelvie, R. S., et al. (2007). Characterization of healthrelated quality of life in heart failure patients with preserved versus low ejection fraction in CHARM. European Journal of Heart Failure, 9, 83-91.

Martens, E. J., Kupper, N., Pedersen, S. S., Aquarius, A. E., \& Denollet, J. (2007). Type D personality is a stable taxonomy in post-MI patients over an 18-month period. Journal of Psychosomatic Research, 63, 545-550.

Masoudi, F. A., Rumsfeld, J. S., Havranek, E. P., House, J. A., Peterson, E. D., Krumholz, H. M., et al. (2004). Age, functional capacity, and health-related quality of life in patients with heart failure. Journal of Cardiac Failure, 10, 368-373.

Mommersteeg, P. M., Denollet, J., Spertus, J. A., \& Pedersen, S. S. (2009). Health status as a risk factor in cardiovascular disease: A systematic review of current evidence. American Heart Journal, 157, 208-218.

Muller-Tasch, T., Peters-Klimm, F., Schellberg, D., Holzapfel, N., Barth, A., Junger, J., et al. (2007). Depression is a major determinant of quality of life in patients with chronic systolic heart failure in general practice. Journal of Cardiac Failure, 13, $818-824$.

Munro, B. (2001). Statistical methods for health care research. Philadelphia: Lippincott Williams \& Wilkins.

Muntwyler, J., Abetel, G., Gruner, C., \& Follath, F. (2002). One-year mortality among unselected outpatients with heart failure. European Heart Journal, 23, 1861-1866.

Pedersen, S. S., \& Denollet, J. (2006). Is Type D personality here to stay? Emerging evidence across cardiovascular disease patient groups. Current Cardiology Reviews, 2, 205-213.

Pedersen, S. S., Denollet, J., Spindler, H., Ong, A. T., Serruys, P. W., Erdman, R. A. M., et al. (2006). Anxiety enhances the detrimental effect of depressive symptoms on health status following percutaneous coronary intervention. Journal of Psychosomatic Research, 61, 783-789.

Pelle, A. J., Denollet, J., Zwisler, A. D., \& Pedersen, S. S. (2009). Overlap and distinctiveness of psychological risk factors in patients with ischemic heart disease and chronic heart failure: Are we there yet? Journal of Affective Disorder, 113, 150-156.

Pelle, A. J. M., Erdman, R. A. M., van Domburg, R. T., Spiering, M., Kazemier, M., \& Pedersen, S. S. (2008). Type D patients experience improved health status following cardiac rehabilitation, but not on par with non-Type D patients. Annals of Behavioral Medicine, 36, 167-175.

Raphael, C., Briscoe, C., Davies, J., Ian Whinnett, Z., Manisty, C., Sutton, R., et al. (2007). Limitations of the New York Heart Association functional classification system and self-reported walking distances in chronic heart failure. Heart, 93, 476-482.

Rector, T. S. (2005). A conceptual model of quality of life in relation to heart failure. Journal of Cardiac Failure, 11, 173-176.

Rector, T. S., Kubo, S. H., \& Cohn, J. N. (1987). Patient's selfassessment of their congestive heart failure. Content, reliability, and validity of a new measure: The Minnesota Living with Heart Failure Questionnaire. Heart Failure, 10, 198-209.

Scherer, M., Stanske, B., Wetzel, D., Koschack, J., Kochen, M. M., \& Herrmann-Lingen, C. (2006). Psychosocial co-symptoms in primary care patients with heart failure. Herz, 31, 347-354.

Schiffer, A. A., Pedersen, S. S., Broers, H., Widdershoven, J. W., \& Denollet, J. (2008a). Type D personality but not depression predicts severity of anxiety in heart failure patients at 1-year follow-up. Journal of Affective Disorders, 106, 73-81.

Schiffer, A. A., Pedersen, S. S., Widdershoven, J. W., \& Denollet, J. (2008b). Type D personality and depressive symptoms are independent predictors of impaired health status in chronic heart failure. European Journal of Heart Failure, 10, 922-930.

Schiffer, A. A., Pedersen, S. S., Widdershoven, J. W., Hendriks, E. H., Winter, J. B., \& Denollet, J. (2005). The distressed (type D) personality is independently associated with impaired health status and increased depressive symptoms in chronic heart failure. European Journal of Cardiovascular Prevention and Rehabilitation, 12, 341-346.

Schiffer, A. A., Smith, O. R., Pedersen, S. S., Widdershoven, J. W., \& Denollet, J. (2009). Type D personality and cardiac mortality in patients with chronic heart failure. International Journal of Cardiology. Jan 20. [Epub ahead of print].

Spertus, J. A. (2008). Evolving applications for patient-centered health status measures. Circulation, 118, 2103-2110.

Whooley, M. (2009). To screen or not to screen? Patients with cardiovascular disease. Journal of the American College of Cardiology, 54, 891-893.

Zigmond, A. S., \& Snaith, R. P. (1983). The hospital anxiety and depression scale. Acta Psychiatrica Scandinavica, 67, 361-370. 